\title{
Temperature-Dependent Field Emission and Breakdown Measurements Using a Pulsed High-Voltage Cryosystem
}

\author{
Marek Jacewicz®, ${ }^{1, *}$ Johan Eriksson $\odot,{ }^{1}$ Roger Ruber, ${ }^{1}$ Sergio Calatroni $\odot,{ }^{2}$ Iaroslava Profatilova $\odot,{ }^{2}$ \\ and Walter Wuensch ${ }^{2}$ \\ ${ }^{1}$ Department of Physics and Astronomy, Uppsala University, Regementsv. 1, 75237 Uppsala, Sweden \\ ${ }^{2}$ CERN, European Organization for Nuclear Research, CH-1211 Geneva 23, Switzerland
}

(Received 1 July 2020; revised 16 September 2020; accepted 3 December 2020; published 30 December 2020)

\begin{abstract}
A variable-temperature pulsed high-voltage system has been constructed and a series of high-field measurements on copper electrodes have been carried out. The measurements are made at ambient to cryogenic temperatures and include conditioning, breakdown threshold, and field emission. A significant, up to 50\%, increase in the breakdown threshold and remarkable stability of field emission are observed when cooled to cryogenic temperatures compared to room temperature. These results provide important experimental input for the development of quantitative theories and models of high-field processes as well as practical input for cryogenic radio-frequency systems.
\end{abstract}

DOI: 10.1103/PhysRevApplied.14.061002

Electron field emission (FE) and vacuum arcing (breakdown) are phenomena that are important for a wide range of applications, including normal conducting and superconducting accelerators. Significant resources have been dedicated by accelerator communities for increasing achievable gradient, including investigations into the fundamental processes that lead to FE and breakdown (BD) [1-5].

In high-gradient accelerator applications, the most important issues include identifying the mechanisms that limit the ultimate surface fields and the origin of the BD rate (BDR), that is, the number of times an accelerating structure breaks down divided by the number of pulses to which it is subject [6,7]. A number of models have been proposed including those of Refs. [8,9], which attribute BD nucleation, and consequently BDR, to lattice void formation and to dislocation dynamics, respectively. In both cases the BDR is predicted to be strongly temperature dependent. Fowler and Nordheim have described the theory behind FE [10]; however, in macroscopic systems a so-far-unidentified field enhancement factor (often denoted as $\beta$ ), of the order of 30 to over 100, must be applied for the theory to fit experiment. Constraining the characteristics of the FE enhancement would be an important contribution to

*marek.jacewicz@physics.uu.se

Published by the American Physical Society under the terms of the Creative Commons Attribution 4.0 International license. Further distribution of this work must maintain attribution to the author(s) and the published article's title, journal citation, and DOI. Funded by Bibsam. the theory. BD nucleation models and the nature of intrinsic field enhancement features can be studied in an original way exploiting temperature dependence, and to this end a dedicated experimental setup has been constructed and high-field measurements have been made in the range from room down to cryogenic temperatures.

The setup is based on a pulsed dc high-field system [11-14], which has been developed to complement the $X$ band radio frequency (rf) test stands of the CLIC study [15]. It consists of planar anode and cathode made of OFE copper in ultrahigh vacuum biased by a kHz-rangecapable, high-voltage (HV) generator [16]. A precisionmanufactured alumina spacer fixes the gap between the electrodes to $62.8 \mu \mathrm{m}$ at $300 \mathrm{~K}$. The electrode system is placed in a cryostat connected to a cryocooler. The electrode assembly is attached to the second stage of the cryocooler with the upper electrode in mechanical contact with the cold finger [17]. The high-field operation, including BD detection, recovery from discharge, and algorithms for conditioning, is derived from $X$-band test facilities [11-14].

Throughout these tests, whenever the system is cooled, intermediate degassing procedures are carried out at critical temperatures of 120,60 , and $30 \mathrm{~K}$ [18]. The procedure consists of: cool-down and stabilization of all surfaces at an intermediate temperature; warming up of electrodes by internal heaters to degas the material; waiting for gases to desorb from the electrodes and condense on the colder outer radiation shield. At each temperature the capacitance of the electrode system is measured and from this the gap distance is estimated to 77,78 , and $79 \mu \mathrm{m}$ at 90,60 , and $30 \mathrm{~K}$, respectively $[19,20]$. 

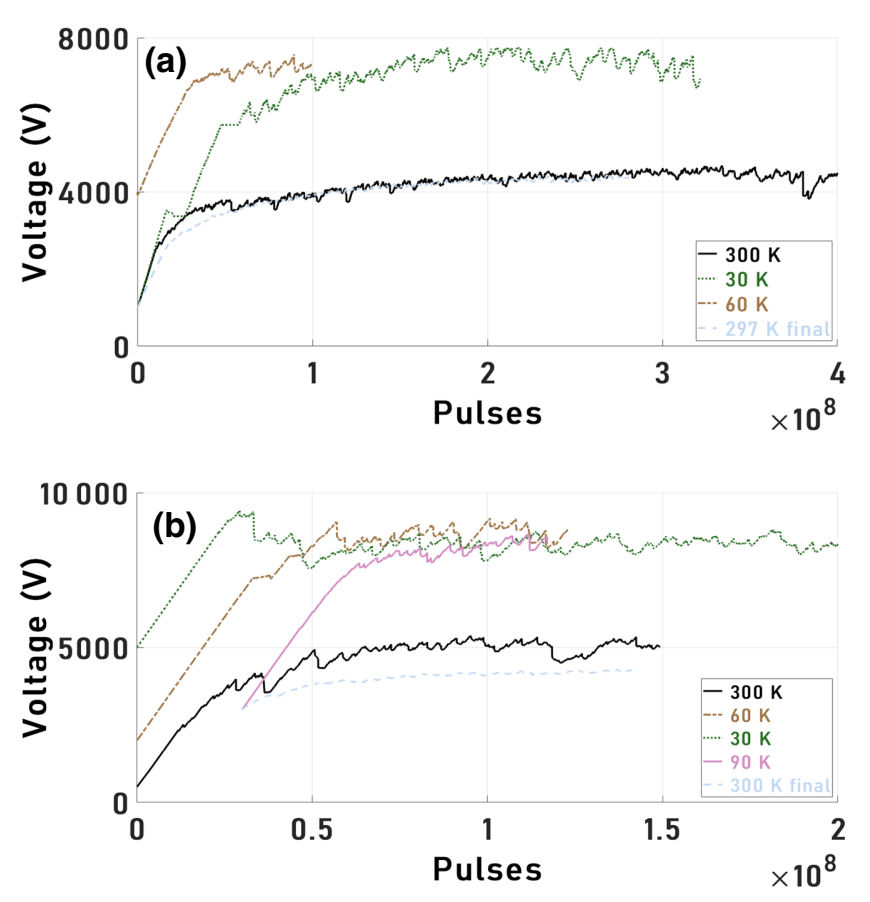

FIG. 1. Conditioning curves for all runs. Data from (a) the first set of electrodes and (b) the second set of electrodes.

Surfaces generally do not initially hold their highest field, and in order to attain it they are subject to progressively higher voltage pulses in a controlled way, a process known as conditioning. In this system, as in the rf structure tests, the pulsed voltage is gradually increased while maintaining a BDR at approximately $10^{-5}$, by feeding back on the ramping rate. The average saturation voltage $V_{\text {sat }}$, calculated by taking an average of $5 \times 10^{6}$ pulses around the maximum voltage, typically occurs within about 100 million pulses and corresponds to stable operation at a BDR of around $10^{-5}$. Details of the conditioning procedure can be found in Refs. $[12,13]$.

After the initial conditioning at $300 \mathrm{~K}$, the first pair of electrodes is conditioned at 30 and $60 \mathrm{~K}$ and the second pair at 30,60, and $90 \mathrm{~K}$. A final conditioning for both pairs is made at room temperature. After each temperature change and at the end of each conditioning run, field emission characteristics are measured.

The conditioning histories for both sets of electrodes, and at all temperatures, are shown in Fig. 1 where the applied voltage is plotted as a function of number of applied pulses.

The conditioning at lower temperatures clearly allows attaining higher saturation voltage levels, and higher saturation fields ( $E_{\text {sat }}=V_{\text {sat }} / d$, where $d$ is the spacing of the electrodes), as shown in Table I. The saturation field has, however, usually been observed to decrease with increasing gap size [12,21,22], and described in terms of $V_{\text {sat }}$ by
TABLE I. Field holding capacity compensated for gap-size effect. Estimated saturation field $E_{\text {sat }}^{\text {est }}$, measured saturation field $E_{\text {sat }}$, and relative change $\Delta E / E$ between them, for conditioning runs at different temperatures $T$.

\begin{tabular}{lcccccccc}
\hline \hline & \multicolumn{3}{c}{ Electrode set 1 } & & \multicolumn{3}{c}{ Electrode set 2 } \\
\cline { 2 - 5 } \cline { 6 - 8 }$T$ & $E_{\text {sat }}^{\text {est }}$ & $E_{\text {sat }}$ & $\Delta E / E$ & & $E_{\text {sat }}^{\text {est }}$ & $E_{\text {sat }}$ & $\Delta E / E$ \\
$(\mathrm{~K})$ & $(\mathrm{MV} / \mathrm{m})$ & $(\mathrm{MV} / \mathrm{m})$ & $(\%)$ & & $(\mathrm{MV} / \mathrm{m})$ & $(\mathrm{MV} / \mathrm{m})$ & $(\%)$ \\
\hline 30 & 67.4 & 96.7 & 43 & & 78.3 & 117.1 & 50 \\
60 & 67.7 & 93.6 & 38 & & 78.6 & 115.0 & 46 \\
90 & $\ldots$ & $\ldots$ & $\ldots$ & & 78.9 & 109.4 & 39 \\
300 & 72.2 & 72.2 & 0 & & 83.9 & 83.9 & 0 \\
\hline \hline
\end{tabular}

the relation

$$
V_{\text {sat }}=k \times d^{0.7},
$$

where the constant $k$ depends on the surface properties and processing procedure of the electrodes.

Using Eq. (1) one can compensate for the effect of the gap increase by calculating the expected $V_{\text {sat }}$ at cryogenic temperatures, taking the $k$ value calculated at room temperature $\left(k_{1}=3.96 \mathrm{MV} / \mathrm{m}^{0.7}\right.$ and $k_{2}=4.61 \mathrm{MV} / \mathrm{m}^{0.7}$, respectively, for the first and second electrode set) and estimating the expected saturation field $E_{\text {sat }}^{\text {est }}$ at gap sizes measured at 90, 60, and $30 \mathrm{~K}$. A comparison of these estimates with the measured values for each of the conditioning runs can be found in Table I. There is a significant increase of the measured saturation field at cryogenic temperatures with respect to room temperature, clearly indicating a strong effect of the cold conditioning. These results are consistent with the experiment performed at SLAC in which a copper accelerating cavity achieved higher rf surface fields for a fixed BDR when operated at cryogenic temperatures [23].

A theoretical model of the BDR dependence on electric field $E$ and temperature $T$, driven by the creation of dislocations under the stress produced by the electric field, described by the activation energy $E_{f}$ and the volume of dislocation nucleation $\Delta V$, was put forward in Ref. [8]. These quantities are related by the equation

$$
T=\frac{-E_{f}+\epsilon_{0} \Delta V E^{2}}{k_{b}(\ln \mathrm{BDR}-\ln a)},
$$

where $\epsilon_{0}$ is the vacuum permittivity, $k_{b}$ is the Boltzmann constant, and $a$ is the prefactor of the Sieverts-type dependence postulated by the model.

We perform a simultaneous least-squared fit to both data sets with parameters described by Eq. (2) keeping $E_{f}$ common for both sets and $\Delta V_{1} / \Delta V_{2}$ and $a_{1} / a_{2}$ different for each set and taking BDR $=10^{-5}$ as fixed by our conditioning algorithm. We obtain the following results: $E_{f}=0.103 \mathrm{eV}$ with $\Delta V_{1}=0.191 \times 10^{-24} \mathrm{~m}^{3}$ and $a_{1}=$ $6.36 \times 10^{-5}$ for the first set of electrodes and $\Delta V_{2}=$ 


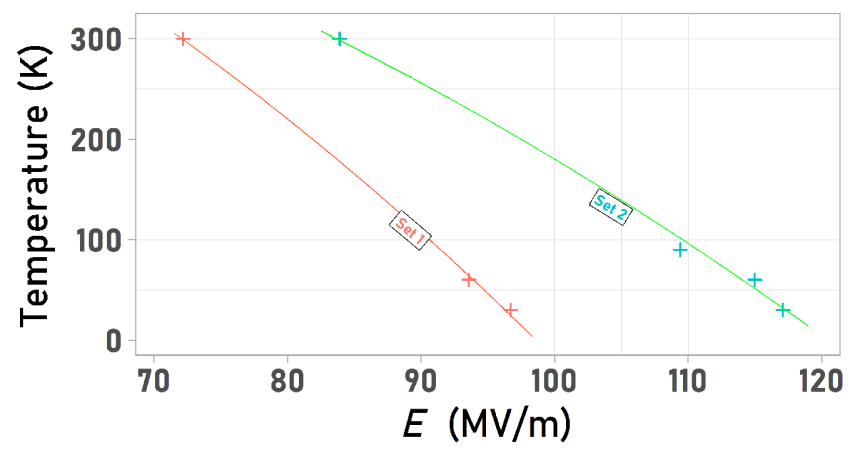

FIG. 2. Measured values of the maximum surface field at different temperatures for both sets of electrodes. The lines are the fits from the crystal defect model [Eq. (2)].

$0.128 \times 10^{-24} \mathrm{~m}^{3}$ and $a_{2}=7.81 \times 10^{-5}$ for the second set. The results are very consistent with the values given in Refs. $[8,24]$. Figure 2 shows the fit of the model to the data. The data are in similar agreement to Ref. [9].

Field emission measurements are carried out after each conditioning run and at each stabilized temperature. Two different instruments are used in the study [25] and provide consistent results. FE current $I$ is measured with constant, that is, not pulsed, applied voltage $V$, which is increased at a rate of $100 \mathrm{~V}$ per minute until maximum voltage is reached or a BD is detected. The possible damage as a result of a BD is significantly limited by available power from the HV power supply. Current-voltage scans are routinely repeated in order to determine if there is any surface degassing of contaminants by electron-stimulated desorption [26] during the measurements, or other effects that might result in a change in the FE properties between the scans. Such a change is observed at room temperature but not at cryogenic temperatures. We believe this confirms that the desorption procedure described before is successful.

FE results relating to different temperatures are presented in the following, plotted as $\ln \left(I / E^{2}\right)$ versus $1 / E$. The Fowler-Nordheim [10] theory predicts a linear behavior in such plots for a single emitter on a perfectly flat surface $[27,28]$. In all the plots dashed lines represent linear fits and solid lines represent a Savitzky-Golay (SG) filter fit to the data [29].

The effect of cooling to cryogenic temperatures can be seen in Fig. 3(a), which shows the examples of FE current scans after conditioning at $300 \mathrm{~K}$ (black curve) and after cooling to $30 \mathrm{~K}$ (green) (but not re-conditioning). The scans in all the cases end with the detection of a BD after reaching on average currents of about $100 \mu \mathrm{A}$. At the lower temperature the current is at least an order of magnitude smaller than at room temperature for the same field value.

The effect of the conditioning at $30 \mathrm{~K}$ is shown in the same figure in brown color. After conditioning at $30 \mathrm{~K}$,
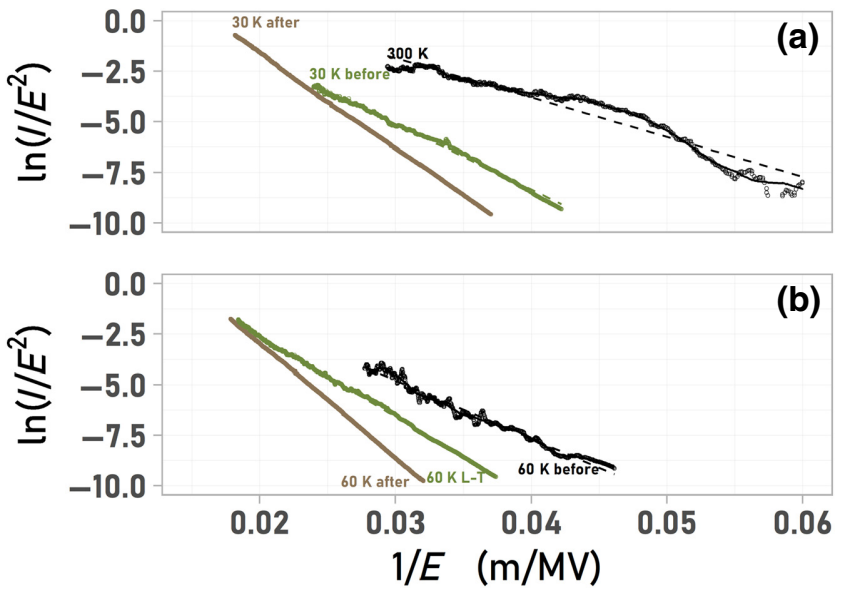

FIG. 3. (a) Field emission after conditioning at $300 \mathrm{~K}$ (black), cool-down to $30 \mathrm{~K}$ (green), and after re-conditioning at $30 \mathrm{~K}$ (brown). (b) FE for surface cooled down to $60 \mathrm{~K}$ after conditioning at $300 \mathrm{~K}$ (black), after re-conditioning at $60 \mathrm{~K}$ (brown), and compared with $\mathrm{FE}$ at $60 \mathrm{~K}$ after 9 days (green).

lower currents are drawn from the surface for the same field; moreover the surface can sustain a higher field resulting in a much higher emitted current, limited only by the power supply ( $3 \mathrm{~mA})$. Most strikingly, the FE current curves become extremely smooth and very linear in the plot.

The same effect is observed at $60 \mathrm{~K}$, which is illustrated in Fig. 3(b) with FE current scans before (black) and after conditioning (brown) at $60 \mathrm{~K}$. The FE curves show very linear behavior throughout the whole scan. We observe the same behavior for both sets of electrodes.

A warm-up test on conditioned surfaces is made to see if the remarkable stability and high level of FE current are maintained when the temperature increases, as shown in Fig. 4. The electrodes conditioned at $30 \mathrm{~K}$ are warmed up to $45 \mathrm{~K}$ and then to $60 \mathrm{~K}$. Subsequently after reconditioning at $60 \mathrm{~K}$ the electrodes are warmed up to 75 and $90 \mathrm{~K}$. In each case the $15 \mathrm{~K}$ difference already reverses the effects of the conditioning. Breakdowns are detected at relatively low voltages and the curves become irregular.Finally a measurement is made to investigate if stable and high-level FE conditions persist in the longer term. After conditioning and measuring $\mathrm{FE}$ at $60 \mathrm{~K}$ the temperature of the system is lowered to $30 \mathrm{~K}$ and left for 9 days. Afterwards the electrodes are warmed up to $60 \mathrm{~K}$, to desorb any gases that might have been adsorbed. The FE measured at $60 \mathrm{~K}$ after 9 days, displayed as a green curve in Fig. 3(b), shows a partial de-conditioning.

A linear fit is performed on the $\ln \left(I / E^{2}\right)$ versus $1 / E$ plots of the FE measurements, and the fitting results are presented in the first three columns in Table II. The emission from the surfaces conditioned at cryogenic temperatures is characterized by much larger values of both intercept and slope, indicating a larger effective emission 

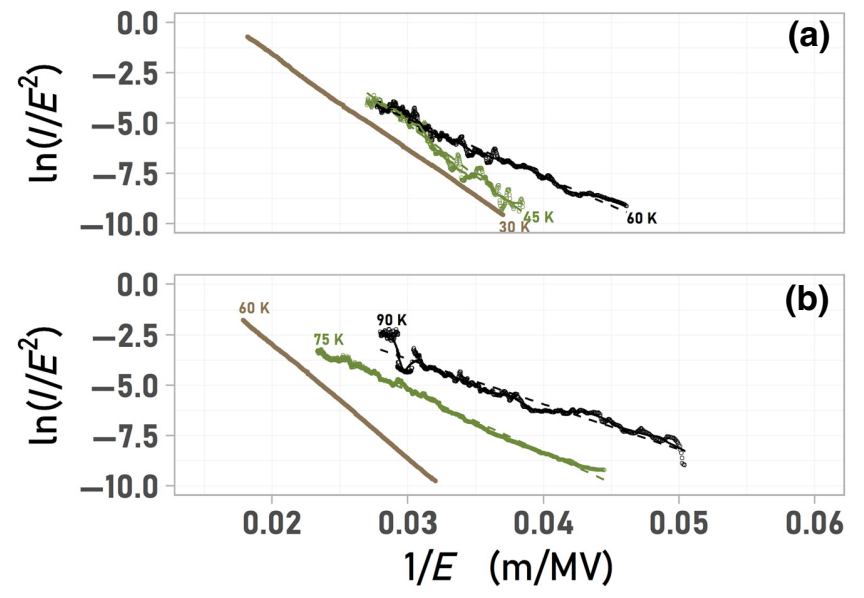

FIG. 4. (a) FE from surfaces conditioned at $30 \mathrm{~K}$ (brown) compared with FE after warm-up to $45 \mathrm{~K}$ (green) and to $60 \mathrm{~K}$ (black). (b) FE from surfaces conditioned at $60 \mathrm{~K}$ (brown) compared with FE after warm-up to $75 \mathrm{~K}$ (green) and to $90 \mathrm{~K}$ (black).

area and a smaller field enhancement as demonstrated by the parameter $\beta$ [28]. A constant work function of copper of $4.65 \mathrm{eV}$ is assumed during the fit of the $\beta$ parameter.

An analysis is made to quantify the noisiness of the FE curves under different conditions, as seen in Figs. 3 and 4, by calculating the deviation from a curve fit to the data. A nonlinear dependence is allowed to accommodate possible distortion of the Fowler-Nordheim curves due to the effects of space-charge or local emitter heating [30]. A SG filter is used for the analysis with three different criteria [31] with the systematic error estimated by repeating the fit with $\pm 10 \%$ variation in SG filter window size. Table II shows the results of the analysis. The three criteria have similar behavior at the different temperatures. The differences after conditioning are striking. For example, at $30 \mathrm{~K}$ the mean square error decreases from $1.7 \%$ to $0.004 \%$, i.e. by a factor 400 , the signal to noise ratio increases by $21 \mathrm{~dB}$, and the smoothing index increases from 0.41 to 0.91 .
Our measurements of the long-term effects of conditioning at cryogenic temperatures (see last row in Table II) indicate that the material and/or surface state changes. After over a week-long interruption we record a change in the slope of the FE curve as well as in all the three parameters describing the smoothness of the FE curves. The change, however, is not as pronounced as the effect of the warm-up of the electrodes.

In conclusion, a cryogenic dc HV system integrated in a stand-alone cryocooler is constructed in order to investigate the fundamental mechanisms of field emission and breakdown nucleation. A series of high-field measurements are carried out with two pairs of copper electrodes at temperatures ranging from ambient down to $30 \mathrm{~K}$. We observe a significant increase in the field holding capability of the electrodes when cooled and conditioned at cryogenic temperatures, with increases of $44 \%$ and $50 \%$ for the two sets at $30 \mathrm{~K}$. The enhancement for measurements at $60 \mathrm{~K}$ decreases by $4 \%-6 \%$ and it further diminishes by $7 \%$ at a temperature of $90 \mathrm{~K}$. The improvement disappears when the surface is warmed up back to room temperature, even after a re-conditioning.

A remarkable reduction of fluctuations of the fieldemitted current from fully conditioned electrodes operated at cryogenic temperatures is also observed, together with an increase of the maximum current enabled by the larger attainable field, consistent with a standard large metal tip theory. The stable and high current field emission quickly deteriorates with increasing temperature, and already with a $15 \mathrm{~K}$ increase the field emission characteristics return to the values from before conditioning.

The results show a general agreement of BD characteristics with the proposed theoretical models, where the temperature enters exponentially via $e^{1 / k_{b} T}$ term $[8,9]$, and confirm the increase in field holding capability at cryogenic temperatures observed in Refs. [23,32,33]. The present work provides experimental data that can be used to refine the theoretical models and potentially

TABLE II. Quantitative comparison of the smoothness of FE curves based on linear regression and Savitzky-Golay fit.

\begin{tabular}{|c|c|c|c|c|c|c|}
\hline Case & Slope $^{\mathrm{a}}$ & Intercept & $\beta$ & $\operatorname{MSE}(\%)^{\mathrm{b}}$ & $\operatorname{SNR}(d B)^{c}$ & $\mathrm{SI}^{\mathrm{d}}$ \\
\hline $300 \mathrm{~K}$ after conditioning at $300 \mathrm{~K}$ & $-184 \pm 2$ & $3.64 \pm 0.1$ & $355 \pm 2$ & $1.2 \pm 0.2$ & $32 \pm 1$ & $0.33 \pm 0.01$ \\
\hline After cool-down to $30 \mathrm{~K}$ & $-279 \pm 2$ & $3.55 \pm 0.1$ & $235 \pm 1$ & $1.7 \pm 0.2$ & $36 \pm 1$ & $0.41 \pm 0.01$ \\
\hline $30 \mathrm{~K}$ after conditioning at $30 \mathrm{~K}$ & $-479 \pm 1$ & $7.99 \pm 0.1$ & $137 \pm 1$ & $0.004 \pm 0.001$ & $57 \pm 1$ & $0.91 \pm 0.01$ \\
\hline After warm-up to $45 \mathrm{~K}$ & $-433 \pm 5$ & $6.54 \pm 0.2$ & $151 \pm 1$ & $2.6 \pm 0.2$ & $38 \pm 1$ & $0.50 \pm 0.01$ \\
\hline After warm-up to $60 \mathrm{~K}$ & $-299 \pm 3$ & $4.08 \pm 0.1$ & $219 \pm 1$ & $2.9 \pm 0.3$ & $32 \pm 1$ & $0.31 \pm 0.01$ \\
\hline $60 \mathrm{~K}$ after conditioning at $60 \mathrm{~K}$ & $-561 \pm 1$ & $8.31 \pm 0.1$ & $117 \pm 1$ & $0.007 \pm 0.001$ & $56 \pm 1$ & $0.92 \pm 0.01$ \\
\hline After warm-up to $75 \mathrm{~K}$ & $-323 \pm 2$ & $4.47 \pm 0.1$ & $203 \pm 1$ & $1.1 \pm 0.1$ & $39 \pm 1$ & $0.37 \pm 0.01$ \\
\hline After warm-up to $90 \mathrm{~K}$ & $-211 \pm 3$ & $2.13 \pm 0.1$ & $310 \pm 2$ & $8.4 \pm 0.7$ & $29 \pm 1$ & $0.39 \pm 0.02$ \\
\hline $60 \mathrm{~K}$ after 9 days & $-387 \pm 1$ & $5.13 \pm 0.1$ & $169 \pm 1$ & $0.124 \pm 0.002$ & $47 \pm 1$ & $0.50 \pm 0.01$ \\
\hline
\end{tabular}

${ }^{a}$ Slope, intercept, and $\beta$ field enhancement factor derived from linear regression fit with standard error on the coefficient.

${ }^{\mathrm{b}}$ Relative mean square error after SG fit.

${ }^{c}$ Signal to noise ratio after SG fit.

d Smoothing index after SG fit. 
discriminate between various underlying physical mechanisms, thus eventually improving our understanding of BD phenomena. Our FE data might also be relevant for benchmarking other theoretical work on nano-tip growth [34], in particular the expectations that these models would have in regards to changes with temperature. We plan to continue with the cryogenic test, and as more temperaturedependent datasets become available the accuracy for theoretical modeling and benchmarking should further improve. We also underline that a temperature variation by an order of magnitude is possible only with a cryogenic experiment like ours, while this would certainly not be feasible upon heating above room temperature, irrespective of any experimental difficulty.

Finally, our study provides crucial data for the design of future normal-conducting accelerators at cryogenic temperatures with very high gradient and reduced breakdown rates [35], with the potential of a reduced cost and optimized performance.

The authors would like to thank Torsten Koettig (CERN) for several helpful discussions and suggestions for the design of the cryogenic system.

[1] W. Wuensch, in 2018 28th International Symposium on Discharges and Electrical Insulation in Vacuum (ISDEIV) (IEEE, Greifswald, Germany, 2018), Vol. 2 p. 747.

[2] G. Ciovati, Review of the frontier workshop and Q-slope results, Physica C: Supercond. 441, 44 (2006).

[3] H. S. Padamsee, Superconducting radio-frequency cavities, Annu. Rev. Nucl. Part. Sci. 64, 175 (2014).

[4] C. E. Reece and G. Ciovati, Superconducting radiofrequency technology R\&D for future accelerator applications, Rev. Accel. Sci. Technol. 05, 285 (2012).

[5] X. Wu, J. Shi, H. Chen, J. Shao, T. Abe, T. Higo, S. Matsumoto, and W. Wuensch, High-gradient breakdown studies of an $x$-band compact linear collider prototype structure, Phys. Rev. Accel. Beams 20, 052001 (2017).

[6] A. Grudiev, S. Calatroni, and W. Wuensch, New local field quantity describing the high gradient limit of accelerating structures, Phys. Rev. ST Accel. Beams 12, 102001 (2009).

[7] A. Degiovanni, W. Wuensch, and J. Giner Navarro, Comparison of the conditioning of high gradient accelerating structures, Phys. Rev. Accel. Beams 19, 032001 (2016).

[8] K. Nordlund and F. Djurabekova, Defect model for the dependence of breakdown rate on external electric fields, Phys. Rev. ST Accel. Beams 15, 071002 (2012).

[9] E. Z. Engelberg, Y. Ashkenazy, and M. Assaf, Stochastic Model of Breakdown Nucleation under Intense Electric Fields, Phys. Rev. Lett. 120, 124801 (2018).

[10] R. H. Fowler and L. Nordheim, Electron emission in intense electric fields, Proc. Roy. Soc. Lond. A119, 173 (1928).

[11] W. Wuensch, A. Degiovanni, S. Calatroni, A. Korsbäck, F. Djurabekova, R. Rajamäki, and J. G. Navarro, Statistics of vacuum breakdown in the high-gradient and low-rate regime, Phys. Rev. Accel. Beams 20, 11007 (2017).
[12] I. Profatilova, W. Wuensch, and S. Calatroni, Behaviour of copper during initial conditioning in the pulse DC system, Tech. Rep. CERN-ACC-2019-0058. CLIC-Note-1145 (CERN, Geneva, 2019).

[13] I. Profatilova, X. Stragier, S. Calatroni, A. Kandratsyeu, E. Rodriguez-Castro, and W. Wuensch, Breakdown localisation in a pulsed dc electrode system, Nucl. Instrum. Methods Phys. Res. Sec. A: Accelerators, Spectrometers, Detectors and Associated Equipment 953, 163079 (2020).

[14] A. Korsback, F. Djurabekova, L. M. Morales, I. Profatilova, E. R. Castro, W. Wuensch, S. Calatroni, and T. Ahlgren, Vacuum electrical breakdown conditioning study in a parallel plate electrode pulsed DC system, Phys. Rev. Accel. Beams 23, 033102 (2020).

[15] P. Burrows, N. Catalan Lasheras, L. Linssen, M. Petri, A. Robson, D. Schulte, E. Sicking, S. Stapnes, eds. The Compact Linear Collider (CLIC) - 2018 Summary Report, CERN Yellow Reports: Monographs (CERN, Geneva, Switzerland, 2018).

[16] L. M. Redondo, A. Kandratsyeu, M. J. Barnes, S. Calatroni, and W. Wuensch, in 2016 IEEE International Power Modulator and High Voltage Conference (IPMHVC) (IEEE, San Francisco, CA, USA, 2016), p. 187.

[17] See Supplemental Material at http://link.aps.org/supplemen tal/10.1103/PhysRevApplied.14.061002 for more details of the setup.

[18] B. Zohuri, Physics of Cryogenics, edited by B. Zohuri (Elsevier, Amsterdam, Netherlands, 2018), p. 1.

[19] See Supplemental Material at http://link.aps.org/supplemen tal/10.1103/PhysRevApplied.14.061002 for more details on the gap size calculations.

[20] G. K. White and M. L. Minges, Thermophysical properties of some key solids: An update, Int. J. Thermophys. 18, 1269 (1997).

[21] A. Maitland, New derivation of the vacuum breakdown equation relating breakdown voltage and electrode separation, J. Appl. Phys. 32, 2399 (1961).

[22] R. P. Little and S. T. Smith, Electrical breakdown in vacuum, IEEE Trans. Electron. Devices 12, 77 (1965), IEEE Transactions on Electron Devices.

[23] A. D. Cahill, J. B. Rosenzweig, V. A. Dolgashev, S. G. Tantawi, and S. Weathersby, High gradient experiments with $X$-band cryogenic copper accelerating cavities, Phys. Rev. Accel. Beams 21, 102002 (2018).

[24] E. Z. Engelberg, A. B. Yashar, Y. Ashkenazy, M. Assaf, and I. Popov, Theory of electric field breakdown nucleation due to mobile dislocations, Phys. Rev. Accel. Beams 22, 083501 (2019), publisher: American Physical Society.

[25] Model Megger MIT525 and Heinzinger HNC 20.000.

[26] A. Descoeudres, Y. Levinsen, S. Calatroni, M. Taborelli, and W. Wuensch, Investigation of the dc vacuum breakdown mechanism, Phys. Rev. ST Accel. Beams 12, 092001 (2009).

[27] T. E. Stern, B. S. Gossling, and R. H. Fowler, Further studies in the emission of electrons from cold metals, Proc. R. Soc. London. Ser. A, Containing Papers Math. Phys. Charact. 124, 699 (1929), publisher: Royal Society.

[28] J. Wang and G. Loew, in Frontiers of accelerator technology. Proceedings, Joint US-CERN-Japan International School, Hayama and Tsukuba, Japan, September 9-18, 1996 (1997) p. 768. 
[29] A. Savitzky and M. J. E. Golay, Smoothing and differentiation of data by simplified least squares procedures, Anal. Chem. 36, 1627 (1964), publisher: American Chemical Society.

[30] W. P. Dyke, J. K. Trolan, E. E. Martin, and J. P. Barbour, The field emission initiated vacuum arc. i. experiments on arc initiation, Phys. Rev. 91, 1043 (1953).

[31] Relative mean square error: $(1 / n) \sum_{i=1}^{n}\left[\left(\hat{x}_{i}-x_{i}\right) / x_{i}\right]^{2}$; signal to noise ratio: $10 \times \log \left[\sum_{i=1}^{n} x_{i}^{2} / \sum_{i=1}^{n}\left(\hat{x}_{i}-x_{i}\right)^{2}\right]$; and smoothing index: $\sum_{i=1}^{n}\left|\hat{x}_{i}+1-\hat{x}_{i}\right| / \sum_{i=1}^{n} \mid x_{i}+1-$ $x_{i} \mid$, where $x_{i}$ and $\hat{x}_{i}$ are data points and points evaluated by the SG filter, respectively.

[32] H. H. Braun, S. Döbert, I. Wilson, and W. Wuensch, Frequency and Temperature Dependence of Electrical Break- down at 21, 30, and 39 ghz, Phys. Rev. Lett. 90, 224801 (2003).

[33] A. Iino, S. Yamaguchi, T. Shintomi, T. Higo, Y. Funahashi, S. Matsumoto, S. Michizono, K. Ueno, and K. Endo, Development of low-loss cryo-accelerating structure with high-purity copper, Nucl. Instrum. Methods Phys. Res. Sec. A: Accelerators, Spectrometers, Detectors and Associated Equipment 866, 40 (2017).

[34] V. Jansson, E. Baibuz, A. Kyritsakis, S. Vigonski, V. Zadin, S. Parviainen, A. Aabloo, and F. Djurabekova, Growth mechanism for nanotips in high electric fields, Nanotechnology 31, 355301 (2020).

[35] J. B. Rosenzweig et al., An ultra-compact $x$-ray freeelectron laser, New. J. Phys. 22, 093067 (2020). 